\title{
SIMULTANEOUS MULTIWAVELENGTH OBSERVATION OF Mkn 501 IN A LOW STATE IN 2006
}

H. Anderhub ${ }^{1}$, L. A. Antonelli ${ }^{2}$, P. Antoranz ${ }^{3}$, M. Backes ${ }^{4}$, C. Baixeras ${ }^{5}$, S. Balestra ${ }^{3}$, J. A. Barrio ${ }^{4}$, D. Bastieri ${ }^{6}$, J. Becerra González ${ }^{7}$, J. K. Becker ${ }^{4}$, W. BednareK ${ }^{8}$, K. Berger ${ }^{8}$, E. Bernardini ${ }^{9}$, A. Biland ${ }^{1}$, R. K. Bock ${ }^{6,10}$, G. Bonnoli ${ }^{11}$, P. Bordas ${ }^{12}$, D. Borla Tridon ${ }^{10}$, V. Bosch-Ramon ${ }^{12}$, D. Bose ${ }^{3}$, I. Braun ${ }^{1}$, T. Bretz ${ }^{13}$, I. Britvitch ${ }^{1}$, M. Camara ${ }^{3}$, E. Carmona ${ }^{10}$, S. Commichau ${ }^{1}$, J. L. Contreras ${ }^{3}$, J. Cortina ${ }^{14}$, M. T. Costado ${ }^{7}, 15$, S. Covino ${ }^{2}$, V. Curtef ${ }^{4}$, F. Dazzi ${ }^{16,28}$, A. De Angelis ${ }^{16}$, E. De Cea del Pozo ${ }^{17}$, R. de los Reyes ${ }^{3}$, B. De Lotto ${ }^{16}$, M. De Maria ${ }^{16}$, F. De Sabata ${ }^{16}$, C. Delgado Mendez $Z^{7,29}$, A. Dominguez ${ }^{18}$, D. Dorner ${ }^{1}$, M. Doro $^{6}$, D. Elsaesser ${ }^{13}$, M. Errando $^{14}$, D. Ferenc ${ }^{19}$, E. Fernández ${ }^{14}$, R. Firpo ${ }^{14}$, M. V. Fonseca ${ }^{3}$, L. Font ${ }^{5}$, N. Galante ${ }^{10}$, R. J. García LóPeZ ${ }^{7}, 15$, M. GarczarczyK ${ }^{14}$, M. Gaug $^{7}$,

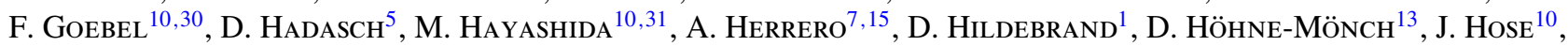
C. C. $\mathrm{Hsu}^{10}$, T. Jogler ${ }^{10}$, D. Kranich ${ }^{1}$, A. La Barbera ${ }^{2}$, A. LaIlle ${ }^{19}$, E. LeONARdo ${ }^{11}$, E. LindFors ${ }^{20}$, S. Lombardi ${ }^{6}$,

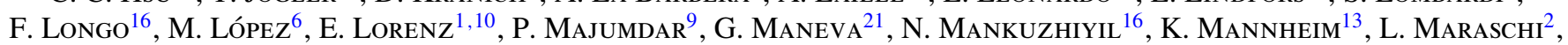
M. Mariotti ${ }^{6}$, M. Martínez ${ }^{14}$, D. Mazin ${ }^{14}$, M. Meucci ${ }^{11}$, M. Meyer ${ }^{13}$, J. M. Miranda ${ }^{3}$, R. Mirzoyan ${ }^{10}$, H. Miyamoto $^{10}$, J. Moldón ${ }^{12}$, M. Moles ${ }^{18}$, A. Moralejo ${ }^{14}$, D. Nieto ${ }^{3}$, K. Nilsson ${ }^{20}$, J. Ninkovic ${ }^{10}$, N. Otte ${ }^{10,32}$, I. Oya ${ }^{3}$, R. PaOletti ${ }^{11}$,

J. M. Paredes ${ }^{12}$, M. Pasanen ${ }^{20}$, D. Pascoli ${ }^{6}$, F. Pauss ${ }^{1}$, R. G. Pegna ${ }^{11}$, M. A. Perez-Torres ${ }^{18}$, M. Persic ${ }^{16,22}$, L. Peruzzo ${ }^{6}$, F. Prada ${ }^{18}$, E. Prandini ${ }^{6}$, N. Puchades ${ }^{14}$, I. ReichardT ${ }^{14}$, W. Rhode ${ }^{4}$, M. Ribó ${ }^{12}$, J. Rico ${ }^{14,23}$, M. Rissi ${ }^{1}$, A. RoberT ${ }^{5}$,

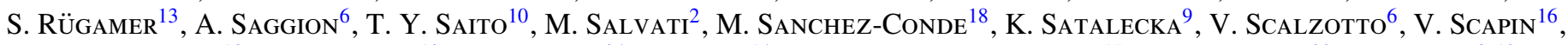
T. Schweizer ${ }^{10}$, M. Shayduk ${ }^{10}$, S. N. Shore ${ }^{24}$, N. Sidro ${ }^{14}$, A. Sierpowska-Bartosik ${ }^{17}$, A. Sillanpää̈ ${ }^{20}$, J. SitareK ${ }^{8,10}$,

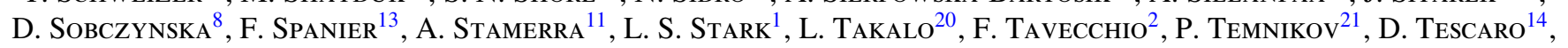

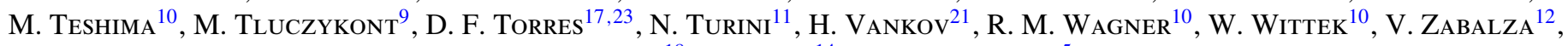
F. ZANDANEL ${ }^{18}$, R. ZANIN $^{14}$, AND J. ZAPATERO ${ }^{5}$

(THE MAGIC COLLABORATION)

AND

R. SAto ${ }^{25}$, M. Ushio ${ }^{25}$, J. KATAOKA ${ }^{26}$, G. MAdEJSKI ${ }^{27}$, AND T. TAKAHASHI ${ }^{25}$

${ }^{1}$ ETH Zurich, CH-8093, Switzerland

${ }^{2}$ INAF National Institute for Astrophysics, I-00136 Rome, Italy

${ }^{3}$ Universidad Complutense, E-28040 Madrid, Spain

${ }^{4}$ Technische Universität Dortmund, D-44221 Dortmund, Germany

${ }^{5}$ Universitat Autònoma de Barcelona, E-08193 Bellaterra, Spain

${ }^{6}$ Università di Padova and INFN, I-35131 Padova, Italy

${ }^{7}$ Inst. de Astrofísica de Canarias, E-38200 La Laguna, Tenerife, Spain

${ }^{8}$ University of Łódź, PL-90236 Lodz, Poland

${ }^{9}$ Deutsches Elektronen-Synchrotron (DESY), D-15738 Zeuthen, Germany

${ }^{10}$ Max-Planck-Institut für Physik, D-80805 München, Germany

${ }^{11}$ Università di Siena, and INFN Pisa, I-53100 Siena, Italy

${ }^{12}$ Universitat de Barcelona (ICC/IEEC), E-08028 Barcelona, Spain

${ }^{13}$ Universität Würzburg, D-97074 Würzburg, Germany

${ }^{14}$ IFAE, Edifici Cn., Campus UAB, E-08193 Bellaterra, Spain

${ }^{15}$ Depto. de Astrofisica, Universidad, E-38206 La Laguna, Tenerife, Spain

${ }^{16}$ Università di Udine, and INFN Trieste, I-33100 Udine, Italy

${ }^{17}$ Institut de Cienciès de l'Espai (IEEC-CSIC), E-08193 Bellaterra, Spain

${ }^{18}$ Inst. de Astrofísica de Andalucia (CSIC), E-18080 Granada, Spain

${ }^{19}$ University of California, Davis, CA-95616-8677, USA

${ }^{20}$ Tuorla Observatory, University of Turku, FI-21500 Piikkiö, Finland

${ }^{21}$ Inst. for Nucl. Research and Nucl. Energy, BG-1784 Sofia, Bulgaria

22 INAF/Osservatorio Astronomico and INFN, I-34143 Trieste, Italy

${ }^{23}$ ICREA, E-08010 Barcelona, Spain

${ }^{24}$ Università di Pisa, and INFN Pisa, I-56126 Pisa, Italy

${ }^{25}$ Institute of Space and Astronautical Science/JAXA, Kanagawa, 229-8510, Japan

${ }^{26}$ Research Institute for Science and Engineering, Waseda University, Tokyo, 169-8555, Japan

${ }^{27}$ Kavli Institute for Particle Astrophysics and Cosmology, SLAC National Accelerator Laboratory, CA, 94025, USA

Received 2009 May 5; accepted 2009 September 28; published 2009 October 26

\begin{abstract}
We present results of the multiwavelength campaign on the TeV blazar Mkn 501 performed in 2006 July, including MAGIC for the very-high-energy (VHE) $\gamma$-ray band and Suzaku for the X-ray band. A VHE $\gamma$-ray signal was clearly detected with an average flux above $200 \mathrm{GeV}$ of $\sim 20 \%$ of the Crab Nebula flux, which indicates a low state of source activity in this energy range. No significant variability has been found during the campaign. The VHE $\gamma$-ray spectrum can be described by a simple power law from $80 \mathrm{GeV}$ to $2 \mathrm{TeV}$ with a photon index of $2.8 \pm 0.1$, which corresponds to one of the steepest photon indices observed in this energy range so far for this object. The X-ray spectrum covers a wide range from 0.6 to $40 \mathrm{keV}$, and is well described by a broken power law, with photon indices of $2.257 \pm 0.004$ and $2.420 \pm 0.012$ below and above the break energy of $3.24_{-0.12}^{+0.13} \mathrm{keV}$. No apparent high-energy cut-off is seen above the break energy. Although an increase of the flux of about $50 \%$ is observed in the X-ray band within the observation, the data indicate a consistently low state of activity for this
\end{abstract}


source. Time-resolved spectra show an evidence for spectral hardening with a flux level. A homogeneous one-zone synchrotron self-Compton (SSC) model can adequately describe the spectral energy distribution (SED) from the X-ray to the VHE $\gamma$-ray bands with a magnetic field intensity $B=0.313 \mathrm{G}$ and a Doppler beaming factor $\delta=20$, which are similar to the values in the past multiwavelength campaigns in high states. Based on our SSC parameters derived for the low state, we are able to reproduce the SED of the high state by just changing the Lorentz factor of the electrons corresponding to the break energy in the primary electron spectrum. This suggests that the variation of the injected electron population in the jet is responsible for the observed low-high state variation of the SED.

Key words: BL Lacertae objects: individual (Markarian 501) - galaxies: jets - gamma rays: observations - X-rays: galaxies

Online-only material: color figures

\section{INTRODUCTION}

Blazars are radio-loud active galactic nuclei (AGNs) viewed at small angles between the jet axis and our line of sight. The bulk relativistic motion of the emitting plasma causes the radiation to be beamed in a forward direction, making the variability appear more rapid and the luminosity appear higher than in the rest frame due to the relativistic beaming effect (e.g., Rees 1966; Ghisellini et al. 1993).

Blazars with only weak or entirely absent emission lines in the optical band are classified as BL Lacertae Objects (BL Lacs). Their spectral energy distributions (SEDs: in $v F_{v}$ ) are characterized by a two-bump structure (Fossati et al. 1998). Since the discovery of the first extra-galactic TeV-photon emitter, Mkn421 (Punch et al. 1992), very-high-energy (VHE; $E>$ $80 \mathrm{GeV}$ ) emission has been confirmed in more than $20 \mathrm{BL}$ Lac objects. The SEDs of many of those BL Lacs show the peaks of the lower energy bump at UV to X-ray energies. These objects belong to the sub-class known as "High-frequency peaked BL Lacs" (HBLs; Padovani \& Giommi 1995). Their nonthermal emission in this lower energy bump is commonly ascribed to synchrotron radiation from relativistic electrons, accelerated in the jet moving with relativistic bulk speed (e.g., Ghisellini et al. 1998). The two-bump structure in SEDs of HBLs has been well explained by synchrotron self-Compton (SSC) models, where the target photons of inverse-Compton scattering for the higher energy bump are the synchrotron photons produced by the same electron population (e.g., Costamante \& Ghisellini 2002). In this model, the high-energy end of the electron spectrum is responsible for both X-ray and VHE $\gamma$-ray emission. The observed correlations of the X-ray and VHE $\gamma$ ray fluxes during large flares of VHE $\gamma$-ray-emitting HBLs (e.g., Takahashi et al. 1996; Maraschi et al. 1999; Krawczynski et al. 2001) provide strong experimental evidence for the SSC mechanism for HBLs. The target photons could also be produced in the accretion disk (Dermer \& Schlickeiser 1993) or in the broadline region (e.g., Sikora et al. 1994). Alternatively, the highenergy emission can be also due to pions produced by accelerated protons and ions and subsequent pion decay (Mannheim 1993) or direct synchrotron emission from high-energy protons (Aharonian 2000).

\footnotetext{
28 Supported by INFN Padova.

${ }^{29}$ Now at: Centro de Investigaciones Energicas, Medioambientales y Tecnologicas (CIEMAT), Madrid, Spain.

${ }^{30}$ Deceased.

${ }^{31}$ Corresponding author, now at: Kavli Institute for Particle Astrophysics and Cosmology, SLAC National Accelerator Laboratory, CA, 94025, USA; mahaya@slac.stanford.edu.

${ }^{32}$ Now at: University of California, Santa Cruz, CA 95064, USA.
}

The results of applying emission models to the data can provide information on physical parameters of the jet, such as the co-moving magnetic field, the population of the accelerated electrons, the Doppler boosting factor, and the size of the emitting region. HBLs often show strong flux variability on timescales of less than $1 \mathrm{hr}$ (Gaidos et al. 1996; Aharonian et al. 2007; Albert et al. 2007b). Hence, simultaneous multiwavelength observations over a wide energy range, covering in particular X-ray and VHE $\gamma$-ray bands, are essential to study the physics of these high-energy radiation emitters.

Until a few years ago, simultaneous multiwavelength observations were only possible during flaring states due to the low sensitivity of the participating $\gamma$-ray telescopes. In the VHE $\gamma$-ray band, new generation of Imaging Atmospheric Cherenkov Telescopes (IACTs), such as MAGIC, H.E.S.S., and VERITAS, can access the energy range from below $100 \mathrm{GeV}$ up to several $\mathrm{TeV}$. These instruments also allow us to detect GeV-TeV $\gamma$ ray signals within short observation times (several hours) even in quiescent source states. A comparison of emission model parameters for several different source states may allow us to reveal the origin of the jet activity.

The Suzaku X-ray satellite (see Section 2.2) features the most sensitive instruments among current X-ray detectors for time-resolved coverage of a wide X-ray energy band, from the soft to the hard X-ray energies, well beyond $10 \mathrm{keV}$. Suzaku already performed observations of several known TeV HBLs and successfully obtained time-resolved spectra up to hard X-ray energies (Sato et al. 2008; Tagliaferri et al. 2008; Reimer et al. 2008). The capability to perform successful multiwavelength observations for TeV-HBLs with MAGIC and Suzaku even in quiescent states has been shown in Tagliaferri et al. (2008) and Reimer et al. (2008).

Mkn 501 (redshift $z=0.034$ ) is categorized as an HBL and was the second established TeV blazar (Quinn et al. 1996). In 1997, this source went into a state of surprisingly high activity. The detected flux was 10 times higher than that of the Crab Nebula in the VHE $\gamma$-ray regime; the high-energy photons were observed up to $\sim 20 \mathrm{TeV}$ (Aharonian et al. 2001). At the same time, BeppoSAX also observed a flaring activity in the X-ray band (Catanese et al. 1997; Pian et al. 1998; Tavecchio et al. 2001). The observed X-ray data showed an exceptionally hard spectrum with a synchrotron peak at (or above) $\sim 100 \mathrm{keV}$. This represents a shift of at least two orders of magnitude with respect to previous observations. Gliozzi et al. (2006) organized a long-term monitoring campaign in 2004, also covering the X-ray and TeV energy bands. They confirm the presence of a direct correlation between X-ray and VHE $\gamma$-ray emission, which appears to be stronger when the source is brighter. In 2005, when MAGIC observed the object between 
May and July, the source flux varied by an order of magnitude. During the two most active nights, rapid VHE $\gamma$-ray flux variability with a doubling time of a few minutes was observed (Albert et al. 2007b). Several extensive SED studies based on multiwavelength observations of this object were reported (e.g., Kataoka et al. 1999; Krawczynski et al. 2000; Sambruna et al. 2000; Tavecchio et al. 2001; Ghisellini et al. 2002). However, the multiwavelength observations that included VHE $\gamma$-ray and $\mathrm{X}$-ray instruments were only conducted during flaring states, but no simultaneous X-ray and VHE $\gamma$-ray data were available for a low state of activity.

In 2006 July, a joint multiwavelength campaign between MAGIC, Suzaku, and the Kungliga Vetenskaplika Academy (KVA) optical telescope ${ }^{33}$ was organized to observe Mkn 501. We succeeded in obtaining clear detections from the simultaneous observations both in the VHE $\gamma$-ray band by MAGIC and in the X-ray band by Suzaku. In this paper, we report the observational results of this campaign. The observations and data reduction for both instruments are briefly described in Section 2. In Section 3, we present the results of the measured light curves and spectra for each energy band. In Section 4, we discuss the application of a simple one-zone SSC model to the SEDs obtained in this campaign and compare those to the historical data from flaring states. Finally, we summarize our results in Section 5.

\section{OBSERVATIONS AND DATA REDUCTION}

\subsection{VHE $\gamma$-ray Band: MAGIC}

The MAGIC telescope is an IACT with a $17 \mathrm{~m}$ diameter dish, located at the Canary Island La Palma (28.2 N, 17.8 W, $2225 \mathrm{~m}$ a.s.1.).

In 2006, Mkn 501 was observed by MAGIC between July and September. The observations were performed in the so-called wobble mode (Daum et al. 1997), where the object is observed with an 0.4 offset from the camera center. With this observation mode, ON- and OFF-data samples can be extracted from the same observation run; in our case, we used three OFF regions to estimate the background. As a part of the multiwavelength campaign, intensive observations were conducted during three nights on July 18,19 , and 20 with a total observation time of $10.5 \mathrm{hr}$. In eight additional nights, MAGIC pointed at this source for only a few tens of minutes each time as a part of an extended monitoring program. In total, $4.2 \mathrm{hr}$ were spent for these additional monitoring observations. After rejecting the data with anomalous trigger rate due to bad observation conditions, the remaining good quality data were further analyzed. Data taken under large zenith angles $\left(>35^{\circ}\right)$ were also excluded to maintain the low energy threshold.

A shower image cleaning was applied based on the charge amplitude and time information in each pixel (Albert et al. 2008a). Every cleaned event was parameterized using the socalled Hillas parameterization (Hillas 1985). These parameters were used for $\gamma /$ hadron separation and energy estimation of $\gamma$-ray events by means of the "Random Forest" (RF) method (Albert et al. 2008b). In the RF method, Monte-Carlo-simulated $\gamma$-ray samples (Majumdar et al. 2005) with the same zenith angle range as the data were used as $\gamma$-ray training sample while real data were used as hadron-event background sample. The $\gamma$-ray signal was extracted on the basis of the DISP method (Domingo-Santamaría et al. 2005); a cut on the $\theta^{2}$ parameter (the

\footnotetext{
33 http://tur3.tur.iac.es/
}

squared angular distance between the nominal source position and the reconstructed $\gamma$-ray direction) was applied to determine the $\gamma$-ray signal (Albert et al. 2007a). Final spectra were derived using an unfolding technique (Albert et al. 2007c). More detailed information on the standard analysis steps and performance of the MAGIC telescope are given in Albert et al. (2008a). As quoted in that paper, we estimate a systematic energy scale error of $16 \%$, a systematic error of $11 \%$ on the flux normalization (without the energy scale error), and a systematic slope error of \pm 0.2 .

For the multiwavelength campaign, based on $9.1 \mathrm{hr}$ of good quality data an excess of 1513 events over 26,112 normalized background events yielding a significance of $8.0 \sigma$ was obtained for the following analysis. We note that tighter cuts that only selected data of a shower image size $>350$ photoelectrons (corresponding to a $\gamma$-ray energy peak of about $250 \mathrm{GeV}$ ) with $\mathrm{a} \theta^{2}<0.03 \mathrm{deg}^{2}$ resulted in an increased $13.4 \sigma$ significance.

\subsection{X-ray Band: Suzaku}

The joint Japanese-US satellite Suzaku (Mitsuda et al. 2007), launched successfully into orbit on 2005 July 10, covers a wide energy range of $0.2-600 \mathrm{keV}$. Suzaku carries four sets of X-ray telescopes (Serlemitsos et al. 2007) each with a focal plane X-ray CCD camera (X-ray Imaging Spectrometer (XIS); Koyama et al. 2007), covering an energy range of 0.3-12 keV. Three of the XIS (XIS 0,2,3) detectors have front-illuminated (FI) CCDs, while the XIS-1 utilizes a back-illuminated (BI) CCD. The merit of the BI CCD is its improved sensitivity in the soft X-ray energy band below $1 \mathrm{keV}$. Suzaku also features a non-imaging collimated Hard X-ray Detector (HXD; Takahashi et al. 2007), consisting of PIN silicon diodes for the lower energy band (10-70 keV) and GSO scintillators for the higher energy band (40-600 keV). Suzaku observations can be conducted using two default pointing positions, the XIS nominal position, and the HXD nominal position. In this observation for the multiwavelength campaign, we used the HXD nominal position to maximize the effective area of the HXD. In the following analysis, the HXD/GSO data are not used because there is no significant detection above the $3 \sigma$ level.

X-ray observations by Suzaku were carried out between 2006 July 18, 18:33:00 UTC and 2006 July 19, 17:27:00 UTC (sequence number 071727010). All XIS sensors were operated with $1 / 4$ window option in order to reduce possible pile-up effects. In total, $35 \mathrm{ks}$ of good time intervals (GTIs) is obtained for each XIS and HXD detector after screening criteria as described in the following.

The XIS data used in this paper were reduced via the Suzaku software version 2.0. The screening was based on the following criteria. (1) ASCA-grade 0,2,3,4, and 6 events were accumulated, and the CLEANSIS script was used to remove hot or flickering pixels. (2) The time interval after the passage of the South Atlantic Anomaly is greater than $500 \mathrm{~s}$. (3) Data were selected to be $5^{\circ}$ in elevation above the rim of the Earth (ELV) (20 above the day-Earth rim). The XIS events were extracted from a circular region with a radius of 4'.2 centered on the source peak, whereas the background was accumulated in an annulus with inner and outer radii 5'.6 and 11'.1, respectively. The response (RMF) and auxiliary files (ARF) are produced using the analysis tools XISRMFGEN and XISSIMARFGEN developed by the Suzaku team (Ishisaki et al. 2007), which are included in the software package HEAsoft version 6.5.

The HXD/PIN data (ver.2.0) were processed with basically the same screening criteria as those for the XIS, except for ELV 

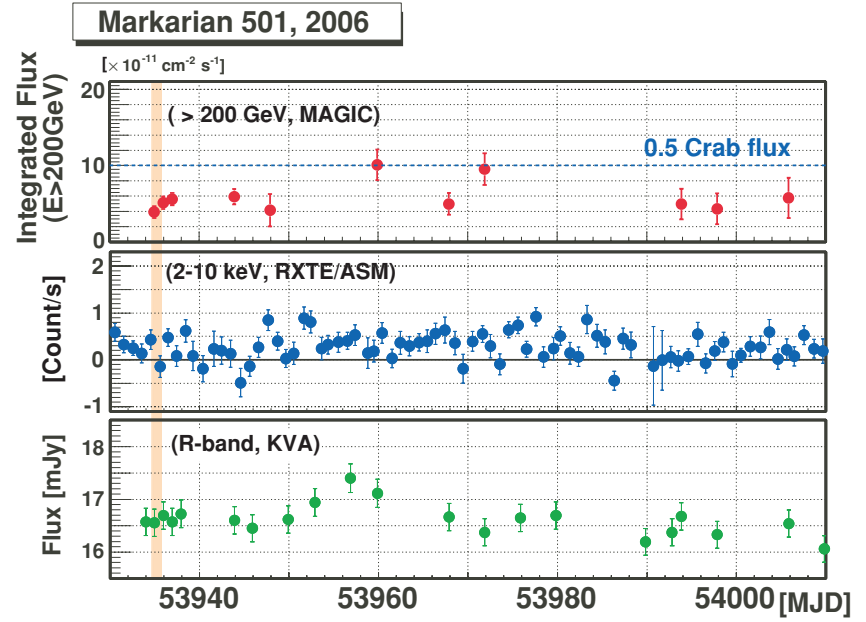

Figure 1. Diurnal multiwavelength light curves during the MAGIC observations of Mkn 501 in 2006 July-September. The vertical band represents the window of the Suzaku pointing. Top: VHE $\gamma$-ray flux above $200 \mathrm{GeV}$ as measured by MAGIC. The horizontal dotted line represents the half flux level of the Crab Nebula in this energy range. Middle: averaged daily X-ray count from RXTE/ ASM. Bottom: optical $R$-band flux by KVA.

(A color version of this figure is available in the online journal.)

$>5^{\circ}$ through night and day and a cut-off rigidity $>8 \mathrm{GeV} / c$. The HXD/PIN instrumental background spectra were provided by the HXD team for each observation (Fukazawa et al. 2006; Kokubun et al. 2007). The HXD/PIN data also include another background component, the so-called, cosmic X-ray background (CXB). In our analysis, we use the CXB spectrum (Gruber et al. 1999) as

$$
\frac{d F}{d E}=9.0 \times 10^{-9}\left(\frac{E}{3 \mathrm{keV}}\right)^{-0.29} \exp \left(\frac{-E}{40 \mathrm{keV}}\right) \frac{\mathrm{erg}}{\mathrm{cm}^{2} \mathrm{~s} \mathrm{srkeV}} .
$$

The observed spectrum was derived assuming the PIN-detector response is isotropic for diffuse emission. Both the source and background spectra were made with identical GTIs, and the exposure was corrected for detector deadtime of $6.0 \%$. We use the response file version ae_hxd_pinhxdnom2_20080129.rsp.

Spectral analysis in the $\mathrm{X}$-ray band was performed using XSPEC version 11.3.2. Each XIS spectrum is binned such that each bin contains at least 40 counts. After the binning, we ignored bins with energies below $0.6 \mathrm{keV}$ and above $10 \mathrm{keV}$. We also excluded bins between 1.7 and $1.9 \mathrm{keV}$ because there exist large systematic uncertainties in the response matrices. Based on a contemporaneous fit to the Crab spectra, Serlemitsos et al. (2007) reported that the normalizations among the CCD sensors are slightly different (by a few \%). A relative normalization of HXD/PIN detector to the CCD cameras also needs to be taken into account (Kokubun et al. 2007). Therefore, the XIS-2, XIS3 , and HXD/PIN spectra were scaled by a constant factor with respect to the XIS-0 spectrum.

\section{RESULTS}

\subsection{Light Curves}

Figure 1 shows the diurnal light curves for Mkn 501 in 2006 with the VHE $\gamma$-ray emission above $200 \mathrm{GeV}$ as measured by MAGIC, public X-ray data taken by $R X T E / \mathrm{ASM}^{34}$ and optical $R$-band data provided by the Tuorla Observatory Blazar

\footnotetext{
34 http://xte.mit.edu/ASM_lc.html
}

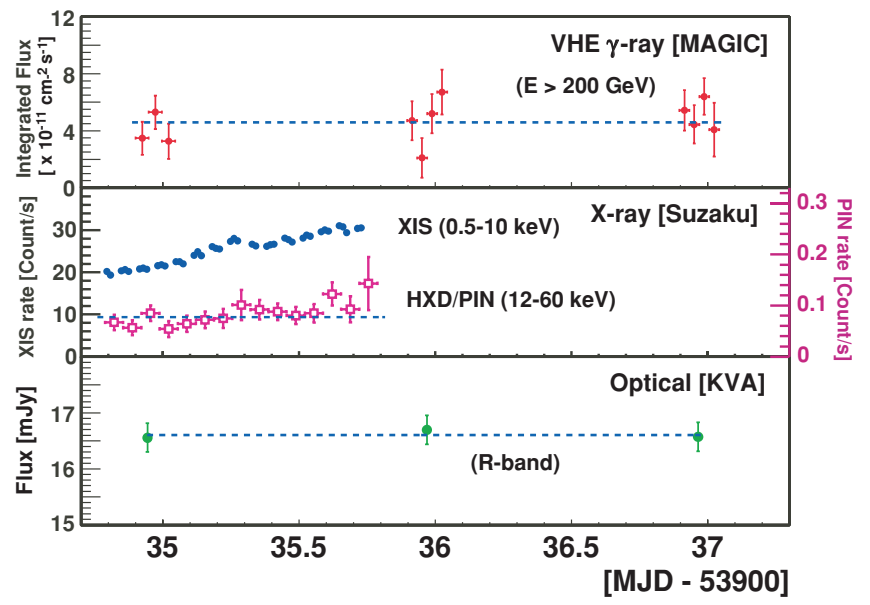

Figure 2. Light curves in different energy bands during this campaign. Each dotted horizontal line represents the average flux for each measurement. Top: VHE $\gamma$-ray flux measured by the MAGIC telescope. Middle: X-ray count rates measured by Suzaku with the four XIS detectors (filled circle) and the HXD/ PIN detector (open square). Bottom: optical $R$-band flux measured by KVA.

(A color version of this figure is available in the online journal.)

Monitoring Program ${ }^{35}$ using KVA. In 2006, the source generally showed a low state of activity in the VHE $\gamma$-ray band, in contrast to 2005 when the flux varied by an order of magnitude up to 3.5 Crab units (Albert et al. 2007b). Such a strong flare activity is not found neither in the X-ray nor the optical bands between 2006 July and September.

The light curves during the multiwavelength campaign in different energy bands taken by MAGIC, Suzaku, and KVA are shown in Figure 2. The binning of the VHE $\gamma$-ray data is $1 \mathrm{hr}$. The average integrated flux above $200 \mathrm{GeV}$ is $(4.6 \pm 0.4) \times$ $10^{-11} \mathrm{~cm}^{-2} \mathrm{~s}^{-1}\left(\chi^{2} /\right.$ d.o.f. $\left.=10.1 / 10\right)$, which corresponds to about $23 \%$ of the Crab Nebula flux as measured by the MAGIC telescope (Albert et al. 2007b). No significant variability is found. However, due to the low source flux level, we could only have seen variability if the flux were to increase by a factor of 2-3.

X-ray count rates of Mkn 501 recorded with the 4 XIS detectors (0.5-10 keV) and the HXD/PIN detector (12-60 keV) are plotted in the middle panel of Figure 2. Each point represents the rate in a time interval of $1440 \mathrm{~s}$ for the XIS and $5760 \mathrm{~s}$ interval for the HXD/PIN. The X-ray count rate in the XIS shows clear variability $\left(\chi^{2} /\right.$ d.o.f. $=2 \times 10^{4} / 41$ for a constant flux fit $)$. It gradually rises during the observation and an overall increase of about $50 \%$ can be seen. The HXD/PIN count rate also seems to follow that increasing trend, but a fit with a constant value yields $\chi^{2} /$ d.o.f. $=13.3 / 14$. The optical $R$-band flux is also consistent with a constant value with an average of $16.6 \pm 0.1 \mathrm{mJy}$.

\subsection{Spectra}

Figure 3 shows the unfolded differential spectrum in the VHE $\gamma$-ray band, averaged over the three days during the campaign. It is well described by a simple power law from $80 \mathrm{GeV}$ to $2 \mathrm{TeV}$ (with $\chi^{2} /$ d.o.f. $=1.85 / 5$ ):

$\frac{d F}{d E}=(1.14 \pm 0.10) \times 10^{-10}\left(\frac{E}{0.3 \mathrm{TeV}}\right)^{-2.79 \pm 0.12} \frac{\text { photons }}{\mathrm{TeV} \mathrm{cm}^{2} \mathrm{~s}}$

\footnotetext{
35 More information at http://users.utu.fi/kani/1m/.
} 


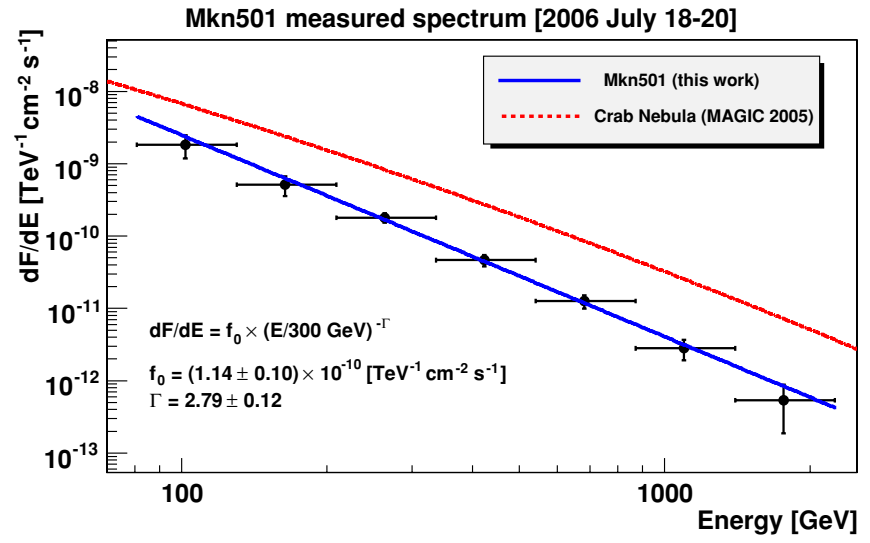

Figure 3. Measured differential energy spectrum at VHE $\gamma$-rays for Mkn 501 with the MAGIC telescope. The blue line corresponds to a simple power-law fit. The fit parameters are listed in the figure. For comparison, the measured MAGIC Crab spectrum (Albert et al. 2008a) is shown as red dashed line. Vertical bars denote the $1 \sigma$ statistical error. Horizontal bars represent the size of the energy bins.

(A color version of this figure is available in the online journal.)

An independent analysis gives results in very good agreement with those quoted numbers. The flux level and the photon index of this spectrum are similar to those in the lowest state among the MAGIC results in 2005 which were presented on a nightby-night basis in Albert et al. (2007b).

Figure 4 shows the Mkn $501 \gamma$-ray spectrum obtained during this multiwavelength campaign together with four spectra measured by MAGIC in 2005 (Albert et al. 2007b) and a spectrum by CAT Cherenkov imaging Telescope on 1997 April 16 (Djannati-Atai et al. 1999). As described in Albert et al. (2007b), the 2005 MAGIC data were subdivided into three groups, i.e., low-, medium- and high states depending on the diurnal integral flux level. The other MAGIC spectrum corresponds to data taken during a strong flare on 2005 June 30 . The CAT data were taken in 1997 during a previous multiwavelength campaign with the BeppoSAX X-ray satellite when the source was in a flaring state. Historically, spectra of Mkn 501 in the VHE $\gamma$-ray band have shown strong variability, and different features of the variability can be seen depending on the energy bands as shown in Figure 4. The difference in flux at $\sim 1 \mathrm{TeV}$ reaches almost two orders of magnitude, while a difference of only a factor of 2-3 in the flux can be seen around $100 \mathrm{GeV}$. The spectrum gets harder as the source flux increases. The energy of the spectral peak, which we consider to be the inverse-Compton peak, can be seen above $100 \mathrm{GeV}$ in the highest flux states (on 1997 April 16 by CAT and on 2005 June 30 by MAGIC), while the peak seems to be located well below $100 \mathrm{GeV}$ in the 2006 observations.

In the $0.6-40 \mathrm{keV} \mathrm{X}$-ray band, we perform a joint fitting with three FI-XISs (XIS-0, 2 and 3: 0.6-10 keV) and HXD/ PIN (12-40 keV) to derive a spectrum. A fixed value of the galactic column density at $1.73 \times 10^{20} \mathrm{~cm}^{-2}$ (Stark et al. 1992) is used for the galactic absorption. The normalization of the $\mathrm{HXD} / \mathrm{PIN}$ with respect to XIS-0 is fixed to be 1.15 as reported in Kokubun et al. (2007). A simple power-law fit yields a photon index of $\Gamma=2.292 \pm 0.002$, but this model gives an unacceptable fit (a reduced $\chi_{v}^{2}=1.107$ with 3893 d.o.f: probability $=2.4 \times 10^{-4} \%$ ). Fitting with a broken power law, we obtain a significantly improved fit (a reduced $\chi_{v}^{2}=1.050$ with 3891 d.o.f: probability $=1.5 \%$ ) with the best-fit photon indices of $\Gamma_{1}=2.257 \pm 0.004$ and $\Gamma_{2}=2.420 \pm 0.012$ below and above the break energy $E_{\text {brk }}=3.24_{-0.12}^{+0.13} \mathrm{keV}$, respectively.

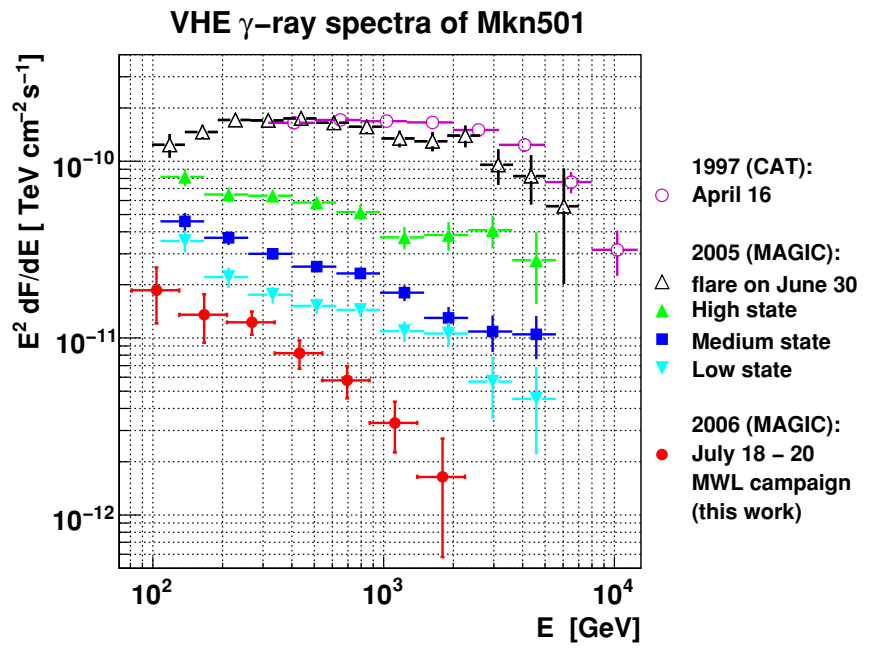

Figure 4. Measured VHE $\gamma$-ray spectra of Mkn 501 in different activity states The CAT data were taken from Djannati-Atai et al. (1999), the 2005 MAGIC data from Albert et al. (2007b), the 2006 MAGIC data from this work. Vertical bars denote the $1 \sigma$ statistical error. Horizontal bars represent the size of the energy bins.

(A color version of this figure is available in the online journal.)

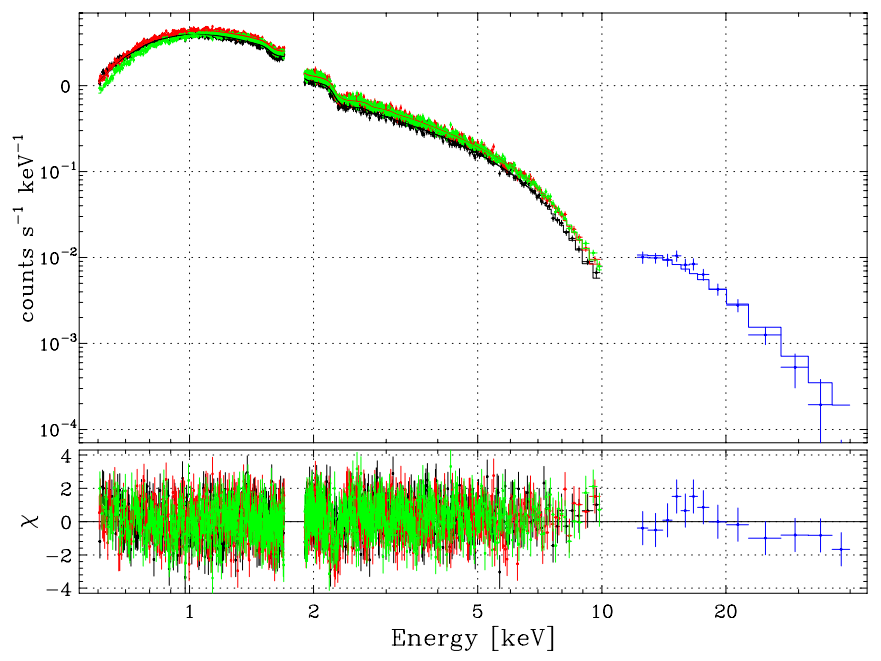

Figure 5. Suzaku (XIS-0 (black), XIS-2(red), XIS-3(green), and HXD/PIN (blue)) averaged spectrum of Mkn 501. The model plotted with the data is a broken power law obtained by a joint fitting to these three XISs and HXD/PIN data. The parameters are shown in Table 1 . The lower panels show the residuals for this broken power-law model. Vertical bars denote the $1 \sigma$ statistical error. (A color version of this figure is available in the online journal.)

The constant factors for XIS-2 and XIS-3 are $1.033 \pm 0.003$ and $0.981 \pm 0.003$, respectively, which are within the values previously reported in Serlemitsos et al. (2007). A doublebroken power-law model does not improve the fit at all. Figure 5 shows background-subtracted folded count spectra of the three FI XISs and HXD/PIN with residuals for the broken power law, using the fixed Galactic column density. The best-fit parameters and associated errors are summarized in Table 1. These derived fit parameters are in good agreement with the results from the XIS data $(0.6-10 \mathrm{keV})$ fit alone. It suggests that there is no significant change (e.g., high-energy cut-off) in the spectrum between $E_{\text {brk }}$ and $40 \mathrm{keV}$. The photon index below the break $\left(\Gamma_{1}\right)$ clearly shows a softer value than 2 . This indicates that the synchrotron peak is located at energies below the XIS range, i.e., lower than $0.6 \mathrm{keV}$.

We also attempted to fit time-resolved broken power-law spectra. The temporal behavior of three parameters, $\Gamma_{1}, \Gamma_{2}$, 
Table 1

Best-fit Parameters for Suzaku Data

\begin{tabular}{|c|c|c|c|c|c|c|c|c|c|c|}
\hline Model & & $\Gamma_{1}$ & $E_{\text {brk }}$ & & $\Gamma_{2}$ & Const. & Const. & Const. & $F_{2-10 \mathrm{keV}}$ & $\chi_{v}^{2} /$ d.o.f. \\
\hline (1) & & (2) & (3) & & (4) & [XIS2] (5) & [XIS3] (6) & {$[\mathrm{HXD} / \mathrm{PIN}](7)$} & (8) & (9) \\
\hline \multicolumn{11}{|c|}{ Suzaku XIS + HXD/PIN } \\
\hline Power law & 2.292 & \pm 0.002 & $\ldots$ & & $\ldots$ & $1.033 \pm 0.003$ & $0.981 \pm 0.003$ & 1.15 (fixed) & $5.370 \pm 0.012$ & $1.107 / 3893\left(2.4 \times 10^{-4} \%\right)$ \\
\hline Broken power law & 2.257 & \pm 0.004 & $3.24_{-0.12}^{+0.13}$ & 2.420 & \pm 0.012 & $1.033 \pm 0.003$ & $0.981 \pm 0.003$ & 1.15 (fixed) & $5.311_{-0.019}^{+0.014}$ & $1.050 / 3891(1.5 \%)$ \\
\hline
\end{tabular}

Galactic column density: $N_{\mathrm{H}}=1.73 \times 10^{20}\left[\mathrm{~cm}^{-2}\right]$ (fixed)

Notes. Column (1): model used to fit the data. Column (2): photon index for the power-law model, or low-energy photon index for the broken power-law model. Column (3): break energy (keV) for the broken power-law model. Column (4): high-energy photon index for the broken power-law model. Columns (5,6,7): constant factors with respect to XIS1, for XIS2, XIS3, and HXD/PIN, respectively. Column (8): Flux in the 2-10 keV band, in units of $10^{-11}$ (erg $\mathrm{cm}^{-2} \mathrm{~s}^{-1}$ ). Column (9): reduced $\chi^{2} /$ degrees of freedom and corresponding probability.

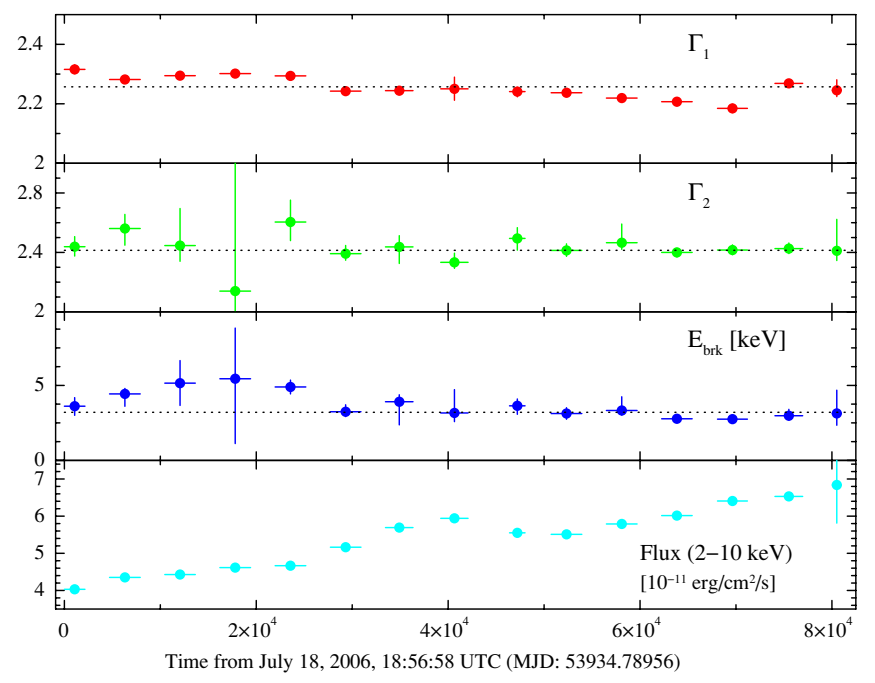

Figure 6. Temporal behavior of the fitting parameters to the XIS data with a broken power-law model. Each point represents a $5760 \mathrm{~s}$ interval. Photon indices below $\left(\Gamma_{1}\right)$ and above $\left(\Gamma_{2}\right)$ the break energy $\left(E_{\text {brk }}\right)$ and model flux between 2 and $10 \mathrm{keV}$ are described. A horizontal dotted line in each panel represents an average value of each parameter. Note that because of a correlation between $\Gamma_{2}$ and $E_{\mathrm{brk}}$, the errors of $\Gamma_{2}$ can also vary depending on the uncertainties of $E_{\mathrm{brk}}$. (A color version of this figure is available in the online journal.)

and the break energy, as well as the model flux between 2 and $10 \mathrm{keV}$, are presented in Figure 6 with a $5760 \mathrm{~s}$ interval. The normalization factors for the XISs were the same as stated above (see Table 1). The model flux increases, similar to the XIS count rate. Figure 7 shows a scatter plot between the model flux between 2 and $10 \mathrm{keV}$ and the photon index $\Gamma_{1}$. A spectral hardening trend by $\sim 0.15$ can be seen in $\Gamma_{1}$ as the flux increases with a correlation coefficient of $r=-\left(0.75_{-0.20}^{+0.02}\right)$. On the other hand, the other parameters do not show strong, flux-dependent variations.

Although the X-ray data show some variability (increase by $\sim 50 \%$ ), given the low flux level, it is still much less than the a factor of $\sim 2$ that would be needed to clearly detect the variability in the VHE $\gamma$-ray range. Thus, in the following section we discuss the broadband SED of Mkn 501 using average spectra in both VHE $\gamma$-ray and X-ray bands taken during this multiwavelength campaign.

\section{DISCUSSION}

Figure 8 shows the overall SEDs of Mkn 501 with data obtained during this multiwavelength campaign as well as some historical data. The flux in the VHE $\gamma$-ray band is corrected for absorption by the extra-galactic background light (EBL) using

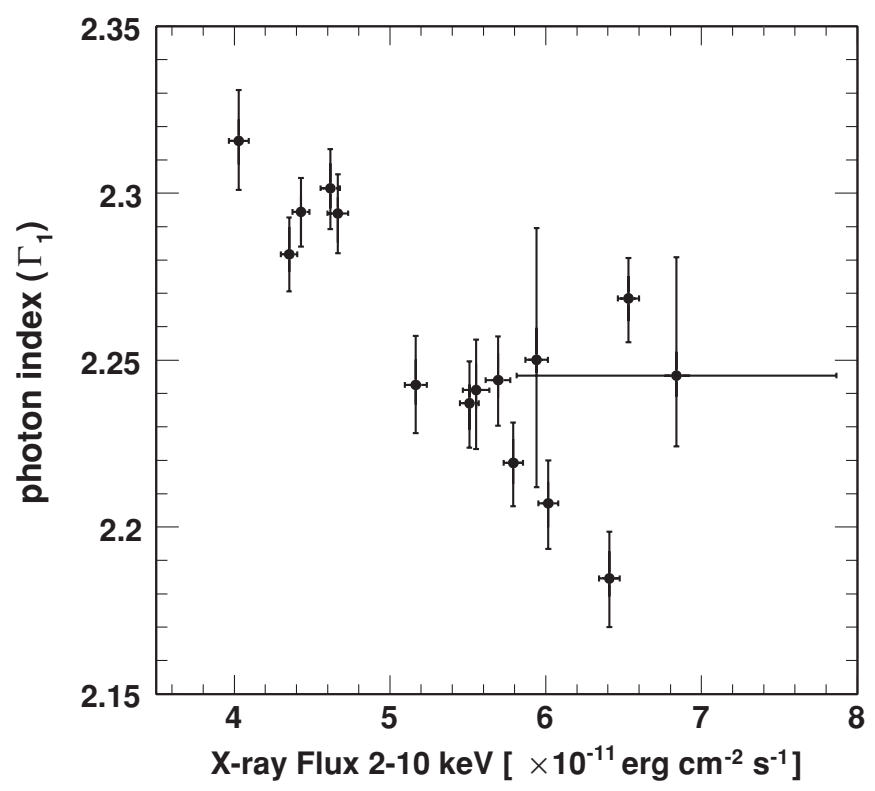

Figure 7. Scatter plot of X-ray flux between 2 and $10 \mathrm{keV}$ and photon index below the break energy $\left(\Gamma_{1}\right)$, which are taken from Figure 6 .

the "low-IR" model of Kneiske et al. (2004). In our optical data, the host galaxy contribution $(12.0 \pm 0.3)(\mathrm{mJy})$ (Nilsson et al. 2007) has already been subtracted.

Assuming a uniform injection of the electrons throughout a homogeneous emission region, we applied a one-zone SSC model, developed by Tavecchio et al. $(1998,2001)$, to our campaign data for estimating physical parameters of the emitting region. A spherical shape (blob) of radius $R$ is adopted for the emission region, filled with a tangled magnetic field of intensity $B$. The electron distribution is described by a smoothed broken power-law energy distribution with slopes $n_{1}$ from $\gamma_{\text {min }}$ to the break energy $\gamma_{\mathrm{b}}$ and $n_{2}$ up to a limit of $\gamma_{\max }$ and with a normalization factor $K$. The relativistic effect is taken into account by the Doppler beaming factor $\delta$.

The radius, $R$, is selected to be $1.03 \times 10^{15} \mathrm{~cm}$, corresponding to the value reported in Albert et al. (2007b) for the SEDs observed in 2005. $\gamma_{\max }$ is set to be $10^{7}$ since no cut-off in the high-energy ends of both the X-ray and the VHE $\gamma$-ray spectra is detected. $\gamma_{\min }$ is fixed at 1 as a nominal value because this parameter does not affect the emission in the energy bands of our data. Since the data do not clearly indicate the positions of the synchrotron and inverse-Compton peaks, we cannot fully constrain the SSC parameters by precise fits to the SEDs. In addition, variability timescales cannot be determined for the data because of the quiescent state of the source. Therefore, our aim 
Table 2

SSC Model Parameters of Mkn 501

\begin{tabular}{lcccccccc}
\hline \hline \multicolumn{1}{c}{ Data } & $\begin{array}{c}R \\
(\mathrm{~cm})\end{array}$ & $\delta$ & $\gamma_{\min }$ & $\gamma_{\text {br }}$ & $\gamma_{\max }$ & $\begin{array}{c}B \\
\text { Gauss }\end{array}$ & $\begin{array}{c}K \\
\left(\mathrm{particle} / \mathrm{cm}^{3}\right)\end{array}$ & $\begin{array}{c}n_{1} \\
n_{2}\end{array}$ \\
\hline 2006 (low) & $1.03 \times 10^{15}$ & 20 & 1 & $6 \times 10^{4}$ & $1 \times 10^{7}$ & 0.313 & $8.5 \times 10^{4}$ & 2 \\
2005 (flare) & $1.03 \times 10^{15}$ & 20 & 1 & $1.3 \times 10^{6}$ & $1 \times 10^{7}$ & 0.313 & $8.5 \times 10^{4}$ & 2 \\
\hline
\end{tabular}

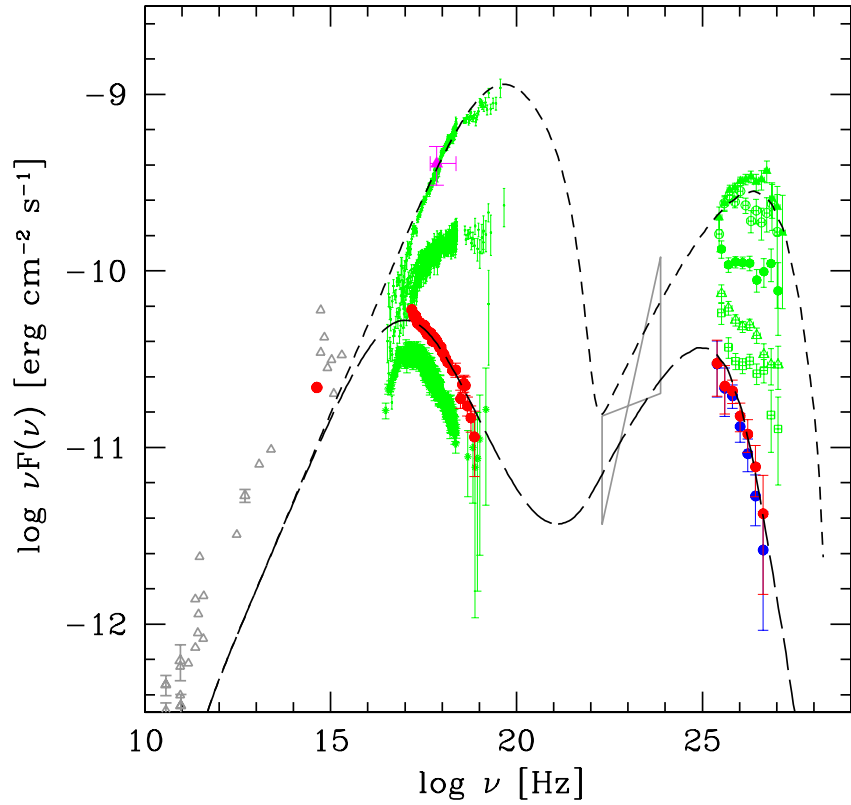

Figure 8. Overall SED of Mkn 501 as measured in 2006 July and historical data. Red points represent energy fluxes from this campaign obtained by KVA (optical), Suzaku (X-ray), and MAGIC (VHE $\gamma$-ray). VHE $\gamma$-ray fluxes are corrected by the "low-IR" EBL model of Kneiske et al. (2004). Corresponding measured VHE $\gamma$-ray fluxes are also plotted by blue points. Green points describe some historical X-ray and VHE $\gamma$-ray fluxes. The X-ray spectra were obtained by BeppoSAX on 1997 April 16 (the highest), 1997 April 29 (medium), and 1999 June (the lowest; taken from Tavecchio et al. 2001). The $\mathrm{X}$-ray flux measured by RXTE/ASM on 2005 Jun 30 is also shown as pink point (see Albert et al. 2007b). The historical VHE $\gamma$-ray spectra were obtained by MAGIC in 2005 taken from Albert et al. (2007b). They are also corrected for EBL absorption using the same Kneiske model. Gray points and a bow-tie are historical data taken from NASA Extragalactic database (radio-optical) and from Kataoka et al. (1999; $\gamma$-ray data observed by EGRET in 1996), respectively. The long-dashed and short-dashed lines describe the SSC model based on Tavecchio et al. $(1998,2001)$ for this campaign data and the "high" state, respectively. The model parameters can be seen in Table 2 .

is to reproduce the observed spectral behavior and correlations within a simple unifying picture rather than entering in details of the jet structure.

First, we applied the SSC model for the low state SED which is obtained during our multiwavelength campaign in 2006. The one-zone SSC model can reproduce the measured X-ray and VHE $\gamma$-ray spectra in this low state of activity of the source as shown in Figure 8. However, it is apparent that the model in Figure 8 underestimates the flux in a low energy range between radio and optical. Usually, homogeneous models cannot be used to explain the low frequency radio emission (see, e.g., Pian et al. 1998) due to efficient self absorption. In previous studies, Katarzynski et al. (2001) used an inhomogeneous conical jet model proposed by Ghisellini et al. (1985) to explain radiation from the low radio frequency up to the ultraviolet of $\mathrm{Mkn}$ 501. Here, along the same lines, we consider the energy range between X-ray and $\mathrm{TeV}$ for our one-zone SSC model.

On the basis of model parameters for this low state, we attempted to reproduce the SED obtained during the flare
Table 3

Comparison of the SSC Model Parameters, $\delta, B$, and $R$ to Previous Studies for Mkn 501

\begin{tabular}{clccc}
\hline \hline$\delta$ & $B(\mathrm{G})$ & $R(\mathrm{~cm})$ & Flux State $^{\mathrm{a}}$ & Reference \\
\hline 15 & 0.8 & $5 \times 10^{15}$ & $\mathrm{H}(1997), \mathrm{M}(1997)$ and L & Pian et al. (1998) \\
15 & 0.2 & $4.5 \times 10^{15}$ & $\mathrm{~L}$ & Kataoka et al. (1999) \\
25 & 0.1 & $4 \times 10^{16}$ & $\mathrm{H}(1998)$ & Sambruna et al. (2000) \\
25 & 0.03 & $2 \times 10^{15}$ & $\mathrm{M}(1998)$ & Sambruna et al. (2000) \\
14 & 0.15 & $2.9 \times 10^{15}$ & $\mathrm{H}(1997)$ & Katarzynski et al. (2001) \\
14 & 0.15 & $4.2 \times 10^{15}$ & $\mathrm{M}(1997)$ & Katarzynski et al. (2001) \\
10 & 0.32 & $1.9 \times 10^{15}$ & $\mathrm{H} \mathrm{(1997),} \mathrm{M} \mathrm{(1997)} \mathrm{and} \mathrm{L}$ & Tavecchio et al. (2001) \\
11 & 0.2 & $10 \times 10^{15}$ & $\mathrm{~L}$ & Kino et al. (2002) \\
50 & 0.04 & $3.5 \times 10^{15}$ & $\mathrm{H} \mathrm{(1997)}$ & Konopelko et al. (2003) \\
20 & 0.313 & $1.03 \times 10^{15}$ & $\mathrm{H} \mathrm{(2005),} \mathrm{L} \mathrm{(2006)}$ & This work
\end{tabular}

Notes.

${ }^{\text {a }} \mathrm{H}$, high state; M, medium state; L, low state (among historical data in X-ray. BeppoSAX (green) data in Figure 8 represent those states). Numbers represent the year when the corresponding data were taken. Previous works for "L" use data taken in different years in different energy bands.

on 2005 June 30, using the same SSC model. There are no simultaneous X-ray data other than RXTE/ASM available at that time. The measured flux by the RXTE/ASM (pink triangle in Figure 8) shows a compatible level in the X-ray spectrum to those taken by BeppoSAX (green dots in Figure 8) on 1997 April 16. In addition, the VHE $\gamma$-ray spectrum taken by MAGIC on 2005 June 30 was almost equivalent to the spectrum measured by CAT on 1997 April 16, as shown in Figure 4. Therefore, we used this BeppoSAX spectrum as a guide for the $\mathrm{X}$-ray spectrum during the VHE $\gamma$-ray flare on 2005 June 30 . With this, we can reproduce the SED in this high state just by changing $\gamma_{\mathrm{b}}$, the Lorentz factor of the electrons at the break energy in the electron spectrum. The SSC models for the low and the high states of Mkn 501 are represented by dashed lines in Figure 8. The derived parameters for these SSC models are listed in Table 2.

In Table 3, we compare our results to some of the previous SED studies based on SSC models for Mkn 501. All of those were derived from applying one-zone SSC models to actual observational data. Not all studies used simultaneous X-ray and VHE $\gamma$-ray data. In fact, most simultaneous X-ray and VHE $\gamma$-ray data were taken during the huge outbursts in 1997. Nevertheless, the SSC model parameters of $\delta$ and $B$ from our multiwavelength campaign in 2006 indicate values similar to those of previous works for different flux states, apart from models of Sambruna et al. (2000; lower $B$ ) for the medium flux state in $1998,^{36}$ and of Konopelko et al. (2003; higher $\delta$ and lower $B$ ) for the high flux state in 1997. Those parameters are also consistent with values in Bednarek \& Protheroe (1999), who constrain the parameters of the emission region based on the variability timescale during the 1997 April 15-16 flaring activity. Note again that only our work used simultaneous X-ray and VHE $\gamma$-ray data taken in a low flux state.

\footnotetext{
${ }^{36}$ However, they did not take into account the absorption by EBL in the VHE $\gamma$-ray data.
} 
Tavecchio et al. (2001) attempted to model different emission states of Mkn 501 in 1997 and 1999 by mainly changing the break energy of electrons, slightly modifying its spectral slopes and number density, and by keeping other parameters unchanged. Also Pian et al. (1998) could reproduce different flux states by changing only the electron distribution, keeping the same values for other parameters. In these frameworks, the electron spectrum is the key component representing the different activity states of Mkn 501; especially, $\gamma_{\text {break }}$ can play a major role there.

From the physical point of view, in the context of the widely discussed diffusive shock acceleration models (Kirk et al. 1998; Henri et al. 1999) the variations of $\gamma_{\text {break }}$ (and the other parameters specifying the particle acceleration) could be explained by changes of the parameters determining the efficiency of the acceleration mechanisms (such as the parameters characterizing the turbulence). A deeper discussion of this point is clearly beyond the scope of this paper.

\section{SUMMARY}

We present first results of a multiwavelength campaign for the TeV blazar Mkn 501 during its low state of activity observed by MAGIC and Suzaku in 2006 July.

1. VHE $\gamma$-ray signals were clearly detected at a $13.4 \sigma$ level from $9.1 \mathrm{hr}$ of data taken during the campaign. The average integrated flux above $200 \mathrm{GeV}$ corresponds to about $23 \%$ of the Crab Nebula flux without significant variability detected in the data. The spectrum in the VHE $\gamma$-ray band is well described by a simple power law from $80 \mathrm{GeV}$ to $2 \mathrm{TeV}$ with a photon index of $2.8 \pm 0.1$. The flux level and the photon index of this measured spectrum are compatible with those found in the lowest state among the MAGIC Mkn 501 observations in 2005.

2. The X-ray spectral shape derived from the Suzaku data from $0.6 \mathrm{keV}$ up to $40 \mathrm{keV}$ is well described by a broken power law without a need for a high-energy cut-off. The derived photon index suggests that the synchrotron peak is located below $0.6 \mathrm{keV}$. The flux level in X-ray showed a low state of activity, in similarity to the VHE $\gamma$-ray flux. As the flux shows a small increase $(\sim 50 \%)$, the spectral index below the break energy shows a hardening trend by $\sim 0.15$.

3 . The overall SED in the low state during our multiwavelength campaign can be well represented by a homogeneous one-zone SSC model. Based on the SSC parameters for this low state, the high-state SED in 2005 can be reproduced by changing only the Lorentz factor of the electrons corresponding to the break energy of the electron spectrum. This suggests that the variation of the injected electron population in the jet is responsible for the observed variation of the SED. In particular, the electron energy at the spectral break could be a key parameter to represent the different activity states of Mkn 501.

We thank the Instituto de Astrofisica de Canarias for the excellent working conditions at the Observatorio del Roque de los Muchachos in La Palma. The support of the German BMBF and MPG, the Italian INFN, and Spanish MCINN is gratefully acknowledged. This work was also supported by ETH Research Grant TH 34/043, by the Polish MNiSzW Grant N
N203 390834, by the YIP of the Helmholtz Gemeinschaft and by the U.S. Department of Energy contract to SLAC no. DEAC3-76SF00515.

\section{REFERENCES}

Aharonian, F. 2000, New Astron., 5, 377 Aharonian, F., et al. 2001, A\&A, 366, 62 Aharonian, F., et al. 2007, ApJ, 664, L71

Albert, J., et al. 2007a, ApJ, 667, L21

Albert, J., et al. 2007b, ApJ, 669, 862

Albert, J., et al. 2007c, Nucl. Instrum. Methods Phys. Res. A, 538, 494

Albert, J., et al. 2008a, ApJ, 674, 1037

Albert, J., et al. 2008b, Nucl. Instrum. Methods Phys, Res. A, 588, 424

Bednarek, W., \& Protheroe, R. J. 1999, MNRAS, 310, 577

Catanese, M., et al. 1997, ApJ, 480, 562

Costamante, L., \& Ghisellini, G. 2002, A\&A, 384, 56

Daum, A., et al. 1997, Astropart. Phys., 8, 1

Dermer, C. D., \& Schlickeiser, R. 1993, ApJ, 416, 458

Djannati-Atai, A., et al. 1999, A\&A, 350, 17

Domingo-Santamaría, E., et al. 2005, Proc. 29th Int. Cosmic Ray Conf. (Pune), 5,363

Fossati, G., Maraschi, L., Celotti, A., Comastri, A., \& Ghisellini, G. 1998, MNRAS, 299, 433

Fukazawa, Y., et al. 2006, Proc. SPIE, 6266, 62662L

Gaidos, J., et al. 1996, Nature, 383, 319

Ghisellini, G., Celotti, A., Fossati, \& Costamante, L. 2002, A\&A, 386, 833

Ghisellini, G., Celotti, A., Fossati, G., Maraschi, L., \& Comastri, A. 1998, MNRAS, 301, 451

Ghisellini, G., Maraschi, L., \& Treves, A. 1985, A\&A, 146, 204

Ghisellini, G., Padovani, P., Celotti, A., \& Maraschi, L. 1993, ApJ, 407, 65

Gliozzi, M., Sambruna, R. M., Jung, I., Krawczynski, H., Horan, D., \& Tavecchio, F. 2006, ApJ, 646, 61

Gruber, D. E., Matteson, J. L., Peterson, L. E., \& Jung, G. V. 1999, ApJ, 520 124

Henri, G., Pelletier, G., Petrucci, P. O., \& Renaud, N. 1999, Astropart. Phys., 11,347

Hillas, A. M. 1985, Proc. 19th Int. Cosmic Ray Conf. (La Jolla), 3, 445

Ishisaki, Y., et al. 2007, PASJ, 59, 113

Kataoka, J., et al. 1999, ApJ, 514, 138

Katarzynski, K., et al. 2001, A\&A, 367, 809

Kino, M., Takahara, F., \& Kusunose, M. 2002, ApJ, 564, 97

Kirk, J. G., Rieger, F. M., \& Mastichiadis, A. 1998, A\&A, 333, 452

Kneiske, T. M., Bretz, T., Mannheim, K., \& Hartman, D. H. 2004, A\&A, 413 807

Kokubun, M., et al. 2007, PASJ, 59, 53

Konopelko, A., et al. 2003, ApJ, 597, 851

Koyama, K., et al. 2007, PASJ, 59, 23

Krawczynski, H., et al. 2000, A\&A, 353, 97

Krawczynski, H., et al. 2001, ApJ, 559, 187

Majumdar, P., et al. 2005, Proc. 29th Int. Cosmic Ray Conf. (Pune), 5, 203

Mannheim, K. 1993, A\&A, 269, 76

Maraschi, L., et al. 1999, Astropart. Phys., 11, 189

Mitsuda, K., et al. 2007, PASJ, 59, 1

Nilsson, K., et al. 2007, A\&A, 475, 199

Padovani, P., \& Giommi, P. 1995, ApJ, 444, 567

Pian, E., et al. 1998, ApJ, 492, L17

Punch, M., et al. 1992, Nature, 358, 477

Quinn, J., et al. 1996, ApJ, 456, L83

Rees, M. J. 1966, Nature, 211, 468

Reimer, A., et al. 2008, ApJ, 682, 775

Sambruna, R. M., et al. 2000, ApJ, 538, 127

Sato, R., et al. 2008, ApJ, 680, L9

Serlemitsos, P. J., et al. 2007, PASJ, 59, 9

Sikora, M., Begelman, M. C., \& Rees, M. J. 1994, ApJ, 421, 153

Stark, A. A., et al. 1992, ApJS, 79, 77

Tagliaferri, G., et al. 2008, ApJ, 679, 1029

Takahashi, T., et al. 1996, ApJ, 470, L89

Takahashi, T., et al. 2007, PASJ, 59, 35

Tavecchio, F., Maraschi, L., \& Ghisellini, G. 1998, ApJ, 509, 608

Tavecchio, F., et al. 2001, ApJ, 554, 725 